\section{Retinal nerve fiber layer thickness in patients with retinitis pigmentosa}

A Oishi, A Otani, M Sasahara, M Kurimoto, H Nakamura, H Kojima, N Yoshimura

\begin{abstract}
Purpose To study retinal nerve fiber layer (RNFL) thickness in patients with retinitis pigmentosa (RP).

Design Cross-sectional observational study. Methods One hundred and thirty-seven eyes of 137 patients with RP were examined. The effect of age, gender, laterality, inheritance trait, spherical equivalent refractive error, visual acuity, and the extent of visual field defect on RNFL thickness measured with optical coherence tomography were analyzed by a multiple linear regression model. Results The average RNFL thickness was $104.1 \pm 21.7 \mu \mathrm{m}$. The multiple $R^{2}$ for the model was 0.349 . Among the variables studied, ageing and being male were significant risk factors for thinner RNFL thickness. RNFL thickness was not correlated with inheritance trait, laterality, refractive error, visual acuity, or the extent of visual field defect.

Conclusion RNFL thickness in RP patients was not correlated with visual function but ageing as in the normal subjects. Currently proposed therapies, including photoreceptor rescue/transplantation and visual prosthesis, are based on the premise that the inner retinal structures are relatively retained despite the profound loss of photoreceptors. The present result supports this notion.
\end{abstract}

Eye (2009) 23, 561-566; doi:10.1038/eye.2008.63; published online 14 March 2008

Keywords: retinitis pigmentosa; retinal nerve fiber; retinal ganglion cell; optical coherence tomography

\section{Introduction}

Retinitis pigmentosa (RP) is a heterogeneous group of hereditary diseases that affects photoreceptors and the retinal pigment epithelium. The disease is a leading cause of blindness with 1.5 million patients in the world. Although intensive research is going on to establish therapeutic approaches for the disease, there is no effective therapy at present.

The currently proposed strategies to restore vision in the disease include delivery of corrective genes or neurotrophic factors, substitutive cell transplantation, and implantation of microelectronics. ${ }^{1}$ However, for successful application of these methodologies in the rescue of photoreceptor function, some preservation of inner retinal structure and function is required. If the inner retinal neurons have died before the therapy, partial rescue or substitute of photoreceptors would fail to restore the visual function. Therefore, whether the inner retina retains its function or not in RP patients is a critical point in considering a novel therapy.

Histopathologic studies have been performed to clarify the inner retinal status of RP patients, showing the preservation of inner retinal cells compared to the almost total loss of photoreceptors; $30-75 \%$ of ganglionic cell-layer cells and up to $90 \%$ of inner nuclear layer cells are retained in the macular area. ${ }^{2,3}$ The percentage decreases to $20-30 \%$ in the ganglion cell layer and approximately $40 \%$ in the inner nuclear layer in the extramacular region. ${ }^{4}$ These studies suggest that inner retinal cells are partially preserved but degenerate with the progression of photoreceptor cell death in RP. The preservation of inner cells was observed in a mouse model of RP as well. ${ }^{5}$ The information is of particular value; however, postmortem analysis cannot be applied for future pretreatment evaluation in each RP case.

Some clinical studies were attempted and showed preservation of optic nerve. Schoth $e^{2} \mathrm{al}^{6}$ examined the optic nerve, -tract, and -radiation with diffusion tensor imaging by MRI, which enables the assessment of cerebral nerve fiber structure and density. They showed the
Department of

Ophthalmology and Visual Sciences, Kyoto University Graduate School of Medicine, Kyoto, Japan

Correspondence:

Dr A Otani,

Department of

Ophthalmology and Visual Sciences,

Kyoto University Graduate School of Medicine, 54, Shougoin Kawahara-cho, Sakyo-ku, Kyoto 606-8507, Japan

Tel: + (81) 75751 3248;

Fax: + (81) 757520933

E-mail: otan@

kuhp.kyoto-u.ac.jp

Received: 15 October 2007 Accepted in revised form:

11 February 2008

Published online: 14 March 2008

Financial interest: None. 
preservation of optic nerve or optic radiation fibers even in the patients who have been blind for 12 years. Morimoto et $a l^{7}$ used transcorneal electrical stimulation and examined the following pupillary response. The method demonstrates the pupillary response in RP patients without light sense indicating remaining pathway between retina and pretectum.

Optical coherence tomography (OCT) is a commercially available noninvasive imaging technology that measures retinal morphology, including the thickness of certain retinal layers, for example, retinal nerve fiber layer (RNFL) thickness. The current version of OCT is equipped with an algorithm to detect the border of RNFL and measure its thickness from the obtained image. The RNFL measurement is widely used in monitoring patients with glaucoma, which primarily affects retinal nerve fibers and ganglion cells. Studies consistently show strong correlation between RNFL thickness and the extent of visual field defect. ${ }^{8,9}$

In the present study, we measured RNFL thickness with OCT and analyzed whether RNFL structure is retained or not in RP patients of various ages and stages of the disease.

\section{Materials and methods}

\section{Subjects}

Patients with RP who visited our institution between 2006 and 2007 were included in the study. RP was diagnosed with the presence of night blindness, characteristic fundus appearance, concentric, ringshaped, or island scotoma, corresponding to the fundus appearance and non-recordable electroretinogram examination.

\section{Exclusion criteria}

Best-corrected visual acuity less than $0.1(20 / 200)$, spherical equivalent refractive error $<-6 \mathrm{D}$, history of cataract surgery, changing refraction more than 2D, diabetes, glaucomatous optic disc, optic nerve head drusen, or other retinal vascular complications. If both eyes of the patient met the criteria, one eye of each patient was randomly included.

The patients included underwent an ophthalmic examination including best-corrected visual acuity measurement, Goldmann perimetry, and RNFL measurements with OCT (Stratus OCT, software version 4.0.1, Carl Zeiss Meditec, Dublin, CA, USA). The examinations were performed within 3-month intervals. OCT measurement was performed with the program Fast RNFL, which scans the retina circumpapillary in a 3.4-mm diameter. The border of RNFL was defined with an edge detection algorithm, which is equipped with the device. (Figure 1) The 12-clock-hour average thickness calculated by the software on OCT was used for the study. When the index of the image intensity was less than seven or there was algorithm failure as indicated by the raster lines that divide the boundaries of the RNFL coming together, the data was excluded from the study.

In assessing visual field, we gave a visual field score for each patient according to the result of Goldmann perimetry. The scoring methods are as follows: isopter used was $\mathrm{V} / 4 \mathrm{e}$, we defined a central 30-degree circle as the central visual field and outside the 40-degree circle as
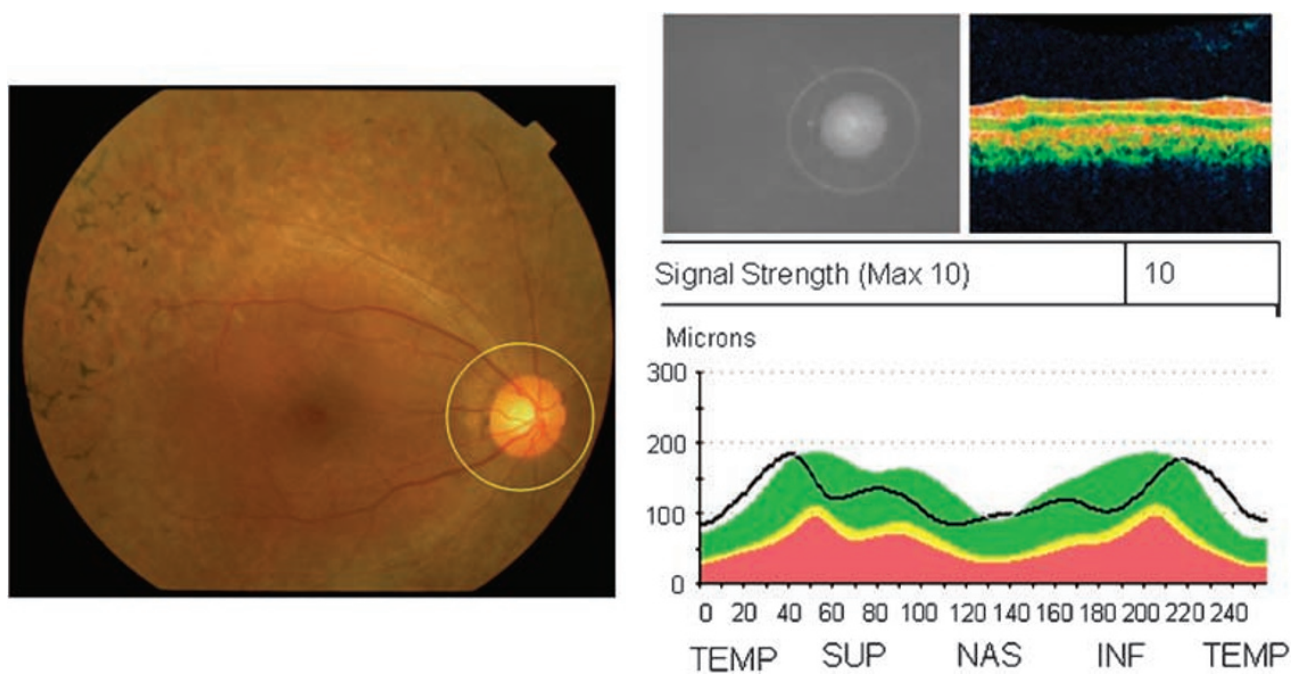

Figure 1 RNFL measurement with OCT in a patient with RP. Left image demonstrates the size and location of 3.4-mm-diameter scanning circle. Scan circle alignment and RNFL border detection was confirmed with right upper image. Right lower image shows RNFL thickness in each segment depicting typical twin peak pattern. 
the peripheral visual field, score 1: central visual field not more than 10 degrees in diameter with no peripheral visual field. 2: central visual field no more than 10 degrees plus peripheral visual field. 3: central visual field $>10$ degrees in diameter but peripheral visual field does not exist. 4: remaining central visual field $>10$ degrees plus peripheral visual field. The details are shown in Figure 2. The retina corresponding to score 1 contains up to $34 \%$ of total number of retinal ganglion cells, whereas score 2: $53 \%$, score 3: $69 \%$, and score $4: 88 \%$, respectively. ${ }^{10}$

\section{Statistical analysis}

The statistical programme SPSS was used to analyse the sample data. Descriptive analyses are reported as mean \pm SD unless otherwise specified. A multiple linear regression analysis of demographic and clinical variables was performed with average RNFL thickness as the dependent variable. The variables used in the model include age, gender, laterality, inheritance trait, and equivalent refractive error. For analysing Inheritance trait, the following numbers were assigned: autosomal recessive: 1 , autosomal dominant: 2 , sporadic: $3, X-$ linked: 4 , and unknown cases: none. The $t$-test was used to compare age and refractive error between genders.

A $P$-value of less than 0.05 was considered statistically significant.

\section{Results}

The study sample consisted of 137 eyes of 60 men and 77 women. Mean age was 50 years. There were 16

Table 1 Demographics of participants

\begin{tabular}{lc}
\hline Age (years) & $50.0 \pm 14.1$ \\
Right/left & $67 / 70$ \\
Sex male/female & $60 / 77$ \\
LogMAR visual acuity (unit) & $-0.19 \pm 0.32$ \\
Spherical equivalent refractive error (diopter) & $-1.36 \pm 2.24$ \\
Visual field score & $2.9 \pm 1.2$ \\
Inheritance trait: autosomal recessive/autosomal & $22 / 32 / 0 /$ \\
dominant/X-linked/sporadic/unknown & $79 / 4$ \\
RNFL thickness $(\mu \mathrm{m})$ & $104.1 \pm 21.7$ \\
\hline
\end{tabular}

Age (years)

$67 / 70$

Sex male/female

$0.19 \pm 0.32$

LogMAR visual acuity (unit)

$1.36 \pm 2.24$

$2 / 32 / 0 /$

$104.1 \pm 21.7$
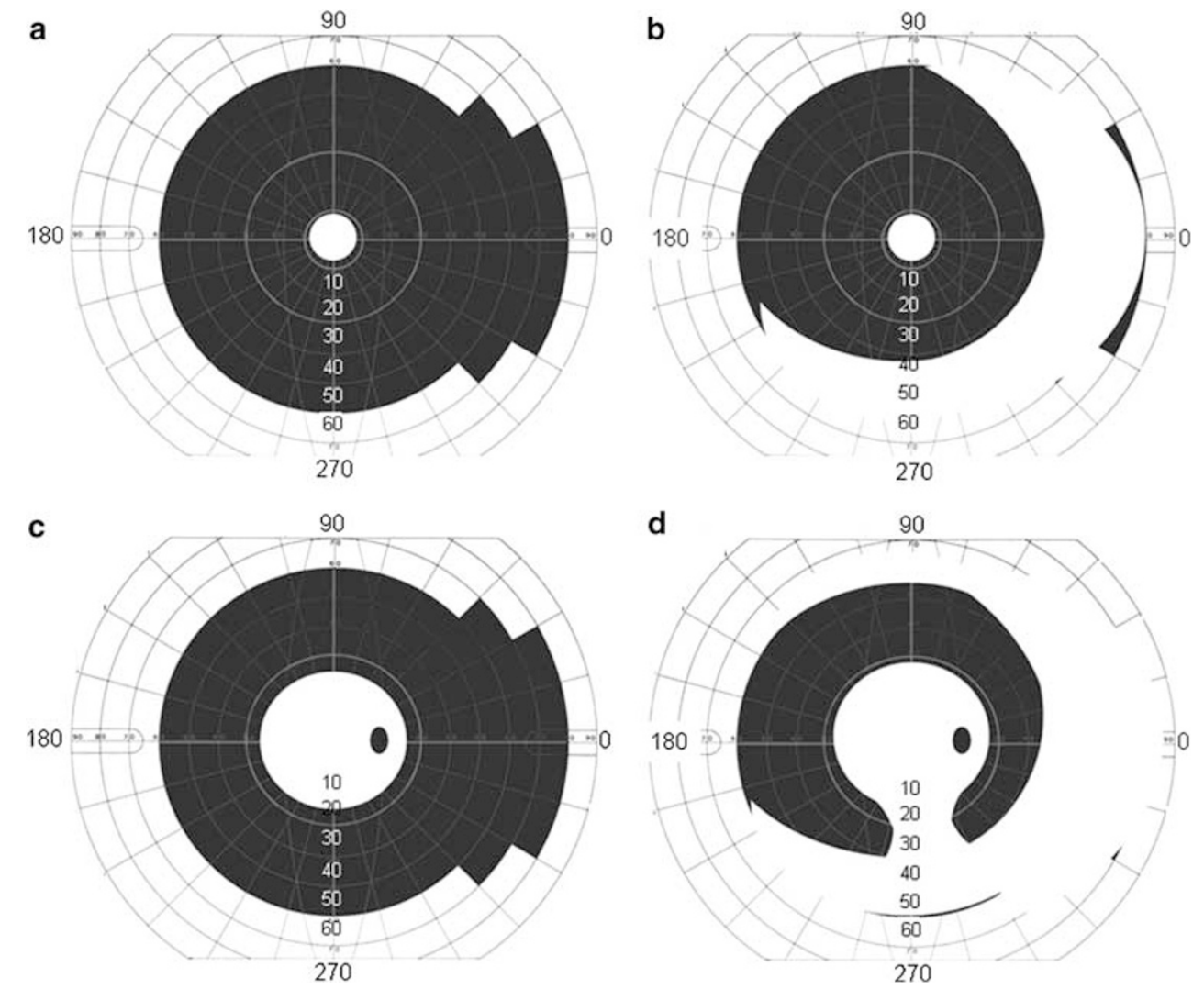

Figure 2 Visual field score criteria. (a) Score 1, central 10-degree diameter without peripheral visual field. (b) Score 2, central 10 degrees with remaining peripheral visual field. (c) Score 3, central visual field extends 10-30 degree but without peripheral visual field. (d) Score 4, central visual field 10-30 degrees with remaining peripheral visual field. 
Table 2 Results of multiple linear regression model of each demographic factor and variable. Multiple $R^{2}$ for the model was 0.346 $(P<0.001)$

\begin{tabular}{|c|c|c|c|c|}
\hline Variable & Regression coefficient & Standard error & P-value & 95\% Confidence interva \\
\hline Age & -0.82 & 0.12 & $<0.001$ & -1.05 to -0.58 \\
\hline Gender & 10.59 & 3.36 & 0.002 & 3.93 to 17.25 \\
\hline Laterality & -4.86 & 3.13 & 0.12 & -11.05 to 1.34 \\
\hline Inheritance trait & -0.55 & 1.86 & 0.77 & -4.23 to 3.14 \\
\hline Refractive error & 0.66 & 0.75 & 0.38 & -0.83 to 2.14 \\
\hline Visual acuity & -0.75 & 5.35 & 0.89 & -11.33 to 9.83 \\
\hline Visual field score & 1.16 & 1.43 & 0.42 & -2.07 to 3.60 \\
\hline
\end{tabular}

pseudophakic eyes. The average RNFL thickness was $104.1 \pm 21.7 \mu \mathrm{m}$. The demographics of the subjects are shown in Table 1.

Table 2 shows the result of a multiple linear regression model. The multiple $R^{2}$ for the model was 0.349 . Among the examined variables, age and gender showed strong correlation. The effects of laterality, inheritance trait, refractive error, visual acuity, and visual field score were not statistically significant. The average RNFL thickness became thinner by approximately $8.3 \mu \mathrm{m}(95 \% \mathrm{CI}, 6.0$ $10.7 \mu \mathrm{m}$ ) for every decade (Figure 3). RNFL of men was thinner than that of women and the difference was approximately $10.9 \mu \mathrm{m}(95 \% \mathrm{CI}, 4.1-17.4 \mu \mathrm{m})$. In this studied population, refractive error was not associated with RNFL thickness.

\section{Discussion}

The aim of this study was to investigate whether OCT-detectable RNFL thickness changes are present in RP patients of various disease severities. Although RP is a disease that primarily affects the photoreceptors, long-standing outer retinal degeneration can induce ascending trans-neuronal degeneration and results in inner retinal cell loss. ${ }^{11}$ Indeed, the typical retinal changes of RP patients, including bone spicule pigmentation and thinning of the retinal vasculature, are located in the inner retinal layer and may represent the degeneration of inner neural components of the retina. The only proven evidence to date regarding how many inner cells are lost is derived from histological analysis of donated eyes. However, in considering therapeutic applications, obtaining the information about the range of degeneration in each RP patient is essential.

OCT enables a noninvasive examination that reveals the detailed structure and specific layer of neural retina. With evolution of technology, the accuracy of OCT has significantly improved and RNFL measurement with OCT is considered one of the most reliable methods to estimate the severity of glaucoma patients to date. ${ }^{12,13} \mathrm{We}$

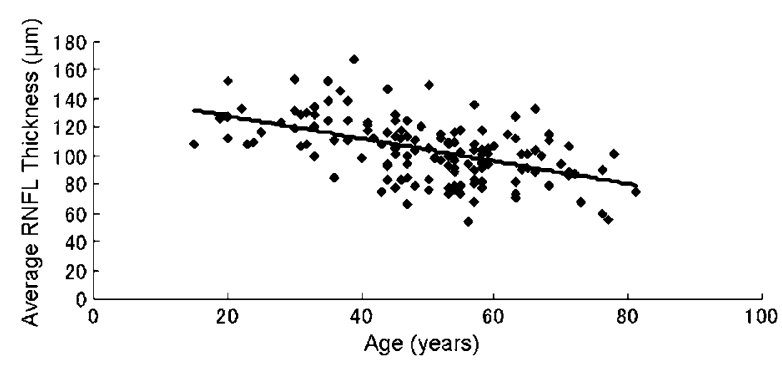

Figure 3 Relationship between RNFL and age in RP patients. Note that RNFL thickness decreases with age at the rate of $8.3 \mu \mathrm{m}$ per decade.

utilized the same method to investigate the RNFL thickness of RP patients.

By analysing the data of 137 eyes, we found that the average RNFL thickness of RP patients $(104.1 \pm 21.7 \mu \mathrm{m})$ was similar to that of healthy Asian subjects $(105.8 \pm 9.2 \mu \mathrm{m}),{ }^{14}$ although certain patients showed thinning of RNFL as reported in very recent study. ${ }^{15}$ In addition, we could find no agreement between RNFL thickness and visual acuity or visual field, that is, the dissociation between structural and functional measures. These data suggest that the photoreceptor death in RP may not easily induce the loss of RNFL and a significant number of inner retinal cells including ganglion cells may survive until the late stage of the disease.

Age is a significant factor for RNFL thickness. RNFL thickness as well as whole retinal thickness of the elderly is known to be thinner than that of the young. ${ }^{16-19}$ In our series, RNFL was thinner in elderly patients than younger patients, as was expected. Interestingly, the rate of RNFL thinning with age was $8.3 \mu \mathrm{m}$ per decade, which was much larger than that of normal subjects, approximately $2 \mu \mathrm{m}$ per decade. ${ }^{14}$ The cause of this phenomenon is not only that the thinning progressed faster in RP patients, but also that the RNFL was thicker in young RP patients and thinner in elder RP patients than normal subjects. Although the mechanisms and meaning of these differences were not investigated in this study, it is possible that the pathological characteristics of 
$\mathrm{RP}$, including inflammatory oedematous thickening of RNFL in young patients, may be involved.

Gender, an unexpected factor on RNFL thickness, showed a strong correlation with RNFL thickness in RP $(P=0.001)$. Male patients had thinner RNFL compared to females. One possible explanation for the finding is the effect of myopia. Some authors report the negative effect of myopia (or axial length) on RNFL thickness. ${ }^{14,20}$ In the present study, the average refractive error of men was smaller than that of women (men: $-2.0 \pm 2.2 \mathrm{D}$, women: $-0.9 \pm 2.2 \mathrm{D}, P=0.002)$, whereas the average age of each group was almost the same (men: $49.8 \pm 14.8$ years women: $50.2 \pm 13.6$ years, non-significant). Refractive error, however, was not a significant predicting factor for RNFL thickness in the present multiple regression analysis, and it is hard to reconcile the large difference between genders. Some factors other than refractive error (ie, inheritance and hormonal circumstance) may exist between gender differences. Anyhow, the result did not show that RP pathology correlates with the RNFL thickness.

One limitation in assessing the result of OCT examination is that the analysis is morphometric but not functional. The study is based on the hypothesis that the retinal thickness represents the volume of retinal nerve fibers and indicates its function. However, there is no evidence that the composition of RNFL measured with OCT in RP patients is the same as that of normal subjects. We cannot exclude the possibility that the atrophic nerve fiber is displaced by fibrous/glial tissue, or residual RNFL exhibits oedematous change simulating false thick RNFL, and ganglion cells are functionally impaired despite morphological preservation. Development of methods to measure the number and function of inner retinal cells including ganglion cell is awaited.

Another limitation includes the subject population. We excluded patients with visual acuity $<0.1$, spherical equivalent $<-6 \mathrm{D}$, or poor image quality to secure the fixation and measurement accuracy. Those with higher disorder were not included in the study because of the inclusion criteria or because we were unable to obtain an analysable image. If these patients were included, the relationship between RNFL thickness and refractive error and visual acuity or visual field analysis could be different.

Finally, we studied RNFL thickness in RP patients with OCT. The result indicated that the retinal nerve fibers are not affected by the degree of the disease, but are lost with ageing, as in normal subjects. The result supports the notion that inner retinal cells are preserved, despite the profound loss of photoreceptors in RP patients and can be the rational for photoreceptor rescuing or substituting therapy. The measurement with OCT is noninvasive, easy to perform, and may determine RNFL loss in patients with RP. Although limitations of OCT measurements exist, further investigation is important in investigating RP pathology.

\section{References}

1 Hartong DT, Berson EL, Dryja TP. Retinitis pigmentosa. Lancet 2006; 368(9549): 1795-1809.

2 Stone JL, Barlow WE, Humayun MS, de Juan Jr E, Milam AH. Morphometric analysis of macular photoreceptors and ganglion cells in retinas with retinitis pigmentosa. Arch Ophthalmol 1992; 110(11): 1634-1639.

3 Santos A, Humayun MS, de Juan Jr E, Greenburg RJ, Marsh MJ, Klock IB et al. Preservation of the inner retina in retinitis pigmentosa. A morphometric analysis. Arch Ophthalmol 1997; 115(4): 511-515.

4 Humayun MS, Prince M, de Juan Jr E, Barron Y, Moskowitz M, Klock IB et al. Morphometric analysis of the extramacular retina from postmortem eyes with retinitis pigmentosa. Invest Ophthalmol Vis Sci 1999; 40(1): 143-148.

5 Gargini C, Terzibasi E, Mazzoni F, Strettoi E. Retinal organization in the retinal degeneration 10 (rd10) mutant mouse: a morphological and ERG study. J Comp Neurol 2007; 500(2): 222-238.

6 Schoth F, Burgel U, Dorsch R, Reinges MH, Krings T. Diffusion tensor imaging in acquired blind humans. Neurosci Lett 2006; 398(3): 178-182.

7 Morimoto T, Fukui T, Matsushita K, Okawa Y, Shimojyo $\mathrm{H}$, Kusaka $\mathrm{S}$ et al. Evaluation of residual retinal function by pupillary constrictions and phosphenes using transcorneal electrical stimulation in patients with retinal degeneration. Graefes Arch Clin Exp Ophthalmol 2006; 244(10): 1283-1292.

8 Harwerth RS, Vilupuru AS, Rangaswamy NV, Smith III EL. The relationship between nerve fiber layer and perimetry measurements. Invest Ophthalmol Vis Sci 2007; 48(2): 763-773.

9 Ajtony C, Balla Z, Somoskeoy S, Kovacs B. Relationship between visual field sensitivity and retinal nerve fiber layer thickness as measured by optical coherence tomography. Invest Ophthalmol Vis Sci 2007; 48(1): 258-263.

10 Rizzo JF. Embryology, anatomy, and physiology of the afferent visual pathway. In: Miller NR, Newman NJ (eds). Walsh \& Hoyt's Clinical Neuro-Ophthalmology, 6th edn: Lippincott Williams \& Wilkins: Philadelphia, 2005, pp 3-82.

11 Gartner S, Henkind P. Pathology of retinitis pigmentosa. Ophthalmology 1982; 89(12): 1425-1432.

12 Budenz DL, Chang RT, Huang X, Knighton RW, Tielsch JM. Reproducibility of retinal nerve fiber thickness measurements using the stratus OCT in normal and glaucomatous eyes. Invest Ophthalmol Vis Sci 2005; 46(7): 2440-2443.

13 Bowd C, Zangwill LM, Medeiros FA, Tavares IM, Hoffmann EM, Bourne RR et al. Structure-function relationships using confocal scanning laser ophthalmoscopy, optical coherence tomography, and scanning laser polarimetry. Invest Ophthalmol Vis Sci 2006; 47(7): 2889-2895.

14 Budenz DL, Anderson DR, Varma R, Schuman J, Cantor L, Savell J et al. Determinants of normal retinal nerve fiber 
layer thickness measured by stratus OCT. Ophthalmology 2007; 114(6): 1046-1052.

15 Walia S, Fishman GA, Edward DP, Lindeman M. Retinal nerve fiber layer defects in RP patients. Invest Ophthalmol Vis Sci 2007; 48(10): 4748-4752.

16 Balazsi AG, Rootman J, Drance SM, Schulzer M, Douglas GR. The effect of age on the nerve fiber population of the human optic nerve. Am J Ophthalmol 1984; 97(6): 760-766.

17 Repka MX, Quigley HA. The effect of age on normal human optic nerve fiber number and diameter. Ophthalmology 1989; 96(1): 26-32.
18 Kanamori A, Escano MF, Eno A, Nakamura M, Maeda H, Seya $\mathrm{R}$ et al. Evaluation of the effect of aging on retinal nerve fiber layer thickness measured by optical coherence tomography. Ophthalmologica 2003; 217(4): 273-278.

19 Alamouti B, Funk J. Retinal thickness decreases with age: an OCT study. Br J Ophthalmol 2003; 87(7): 899-901.

20 Leung CK, Mohamed S, Leung KS, Cheung CY, Chan SL, Cheng DK et al. Retinal nerve fiber layer measurements in myopia: An optical coherence tomography study. Invest Ophthalmol Vis Sci 2006; 47(12): 5171-5176. 